\title{
USO DE HIDRATACIÓN EN DEPORTISTAS RECREATIVOS DE GIMNASIOS DE VIÑA DEL MAR
}

\section{HIDRATION IN RECREATIVE ATHLETS OF GYMNASIUMS OF VIÑA DEL MAR}

\author{
Escobar Matus, Adrián; Pavez Ramírez, Francisco; Prieto Orellana, Pamela; Valdivia Orrego, Jocelyn. \& Crovetto \\ Mattassi, Mirta
}

Nutrición y Dietética, Facultad de Ciencias Naturales y Exactas, Universidad de Playa Ancha, Chile.

ESCOBAR, M.A.; PAVEZ, R.F.; PRIETO, O.P. \& VALDIVIA, O.J. \& CROVETTO, M.M. Uso de hidratación en deportistas recreativos de gimnasios de Viña del Mar. Rev. Motr. Hum., 10(2):49-53, 2009.

\begin{abstract}
RESUMEN
La hidratación al momento de hacer ejercicio resulta indispensable, esta acción puede modificar el rendimiento físico debido a que el consumo de líquido favorece la termorregulación. La falta de ingesta de fluidos perjudica el mantenimiento de la temperatura corporal central, alterando la función cardiovascular y cognitiva y, en algunos casos, provocando la muerte por deshidratación o por el llamado "golpe de calor". Se evaluó a un total de 127 usuarios de gimnasios de las comunas de Valparaíso y Viña del Mar, 39 sujetos del sexo femenino y 88 sujetos del sexo masculino. Los sujetos fueron voluntarios que aceptaron participar de la encuesta y que asistían entre 3 a 5 veces por semana a sesiones de entre 1 a 2 horas de ejercicio por sesión en los respectivos gimnasios. Los sujetos se hidratan principalmente motivados por el mecanismo de la sed, cuando ya existe una pérdida de líquido importante que perjudica el rendimiento. No existe el conocimiento adecuado de los beneficios que otorga una hidratación oportuna o las posibilidades de hidratarse con líquidos isotónicos o enriquecidos con carbohidratos, que favorecen la disponibilidad de energía, prolongando el tiempo de ejecución del ejercicio.
\end{abstract}

Palabras Clave: Hidratación, Deshidratación, Actividad Física.

\section{INTRODUCCIÓN}

La hidratación al momento de hacer ejercicio resulta indispensable, esta acción puede modificar el rendimiento físico debido a que el consumo de líquido favorece la termorregulación $(1,2)$. La falta de ingesta de fluidos perjudica el mantenimiento de la temperatura corporal central, alterando la función cardiovascular y cognitiva $(3,4,5) \quad y$, en algunos casos, provocando la muerte por deshidratación o por el llamado "golpe de calor" (6).

Edward Coyle demostró que la deshidratación en ciclistas de resistencia producía una disminución del flujo sanguíneo a la piel, lo que provocaba un aumento de la temperatura corporal central, aumento de la frecuencia cardiaca y una disminución del volumen sistólico (7).

Al realizar ejercicio, el mecanismo de la sed no es suficiente para mantener un estado de hidratación normal, ya que la pérdida de agua se ha iniciado al aparecer la sed (8). Con un estado de hipohidratación cercano al 2\%, aparece el mecanismo de la sed, donde ya existe deterioro del desempeño físico (9).

El sudor está compuesto principalmente por agua (99\%), pero puede contener algunos electrolitos y micronutrientes importantes. El sudor es hipotónico con respecto a otros líquidos corporales, es decir, tiene un contenido menor de electrolitos (10).

Los principales componentes del sudor son sodio y cloro, que el sudor deriva esencialmente de los líquidos intersticiales que tienen estos componentes. Al unirse, ambos forman el cloruro de sodio, conocido normalmente como sal, la cual se pierde en promedio 2,6 gramos por litro de sudor (11).

La reposición de los elementos perdidos durante las actividades físicas y en situación de calor, resulta fundamental para el óptimo funcionamiento homeostático de nuestro organismo. Según Ron Maughan, estos líquidos 
reponedores deben tener tres elementos principales: agua, carbohidratos y electrolitos (12). A pesar de ello, es posible reemplazar la cantidad de sodio perdida durante el ejercicio con la simple ingesta de agua, ya que los líquidos corporales quedan hipertónicos con el sudor, por lo que el reemplazo no es tan importante (13).

Pero poco sirve confirmar que una completa hidratación nos ayudará en el rendimiento, en el aumento de la capacidad de ejercicio o en el mantenimiento de la homeostasis; si los deportistas no consideran este factor como importante. De acuerdo a esto, revisaremos las conductas de hidratación de un grupo de deportistas recreativos y amateur que asisten a gimnasios en la Quinta Región costa y podremos conocer la situación actual en cuanto a posibles opciones de compra, obtención y conocimiento sobre la hidratación o rehidratación de estos sujetos.

\section{SUJETOS Y MÉTODOS}

Se evaluó a un total de 127 usuarios de gimnasios de las comunas de Valparaíso y Viña del Mar, 39 sujetos del sexo femenino y 88 sujetos del sexo masculino. Los sujetos fueron voluntarios que aceptaron participar de la encuesta y que asistían entre 3 a 5 veces por semana a sesiones de 1 a 2 horas en los respectivos gimnasios.

Estos gimnasios fueron escogidos bajo un método aleatorio simple, de acuerdo a la aparición en el registro telefónico.

Se aplicó una encuesta sobre los hábitos de hidratación que realizaban en sus jornadas de ejercicio, donde se les preguntó si se hidrataban al hacer ejercicio, en qué momento lo hacían, que tipo de líquido utilizaban, con qué objetivo se hidrataban y si daban cuenta de conocer la diferencia entre las bebidas deportivas y las bebidas energéticas.

Las encuestas fueron previamente validadas aplicándolas a un grupo de 30 estudiantes universitarios de Educación Física, realizando las correcciones necesarias y aplicándolas, nuevamente, a otro grupo de estudiantes de Educación Física. La encuesta contiene 6 preguntas de tipo cerrada con alternativas para responder y una pregunta abierta final.

Los resultados de la encuesta se analizan en una aplicación Excel 2007 de Microsoft, realizando una estadística simple.

\section{RESULTADOS}

Se tratarán los resultados más importantes de la encuesta y no se diferenciarán entre el grupo masculino y femenino por lo reducido de la muestra.

Del total de los sujetos encuestados, el $83 \%$ del total indica hidratarse al hacer ejercicio, el $12 \%$ a veces y el $5 \%$ no se hidrata.

A la pregunta de "cuándo se hidrata", las respuestas son variadas, pero la mayoría de los individuos, equivalente a un $43 \%$ de la muestra, señala hidratarse antes, durante y después del ejercicio (Figura 1).

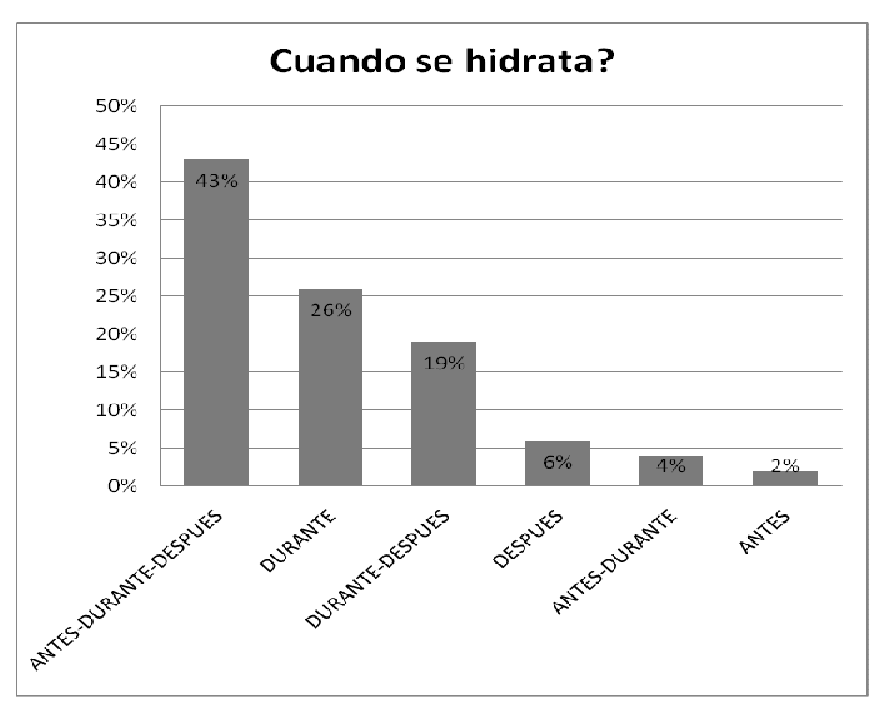

Figura 1: Distribución de las respuestas a la pregunta, ¿Cuándo se hidrata? Clasificación en porcentajes del total de la muestra.

De acuerdo al tipo de líquido que utilizan, un gran porcentaje de los sujetos manifiesta hidratarse con agua (62\%) y un $37 \%$ con bebidas deportivas (Tabla 1). 
¿Qué tipo de líquido utiliza?

\begin{tabular}{ccccccc} 
& AGUA & GATORADE & POWERADE & GO & OTRAS & ENERGÉTICAS \\
\hline$N^{\circ}$ de Sujetos & 85 & 10 & 5 & 31 & 6 & 1 \\
$\%$ de Sujetos & $62 \%$ & $4 \%$ & $7 \%$ & $22 \%$ & $4 \%$ & $1 \%$
\end{tabular}

Tabla 1: Número de sujetos y sus porcentajes del total de la muestra, de acuerdo al tipo de líquido que utilizan para hidratarse.

Otra de las preguntas importantes es respecto al motivo por el cual se hidrataban, donde el $41 \%$ señala hacerlo por sed, el $18 \%$ para tener energía y un $41 \%$ para la sed y tener energía.

Una última pregunta del tipo abierta, pretendía conocer si los usuarios conocían la diferencia entre las bebidas deportivas y las bebidas energéticas, donde el $64 \%$ de los sujetos indica no conocer la diferencia y un $36 \%$ si conoce los elementos que diferencias ambos tipos de bebidas.

\section{DISCUSIÓN}

En esta muestra se pone de manifiesto que la principal forma de hidratarse es por medio de agua, que es un líquido hipotónico con una concentración de partículas por unidad de volumen inferior a la del plasma sanguíneo (menor presión osmótica).

Durante una hora de ejercicio intenso se puede perder hasta 3 litros de líquido, de los cuales 3,5 gramos son de sodio $(14,15)$; por lo tanto, es importante la reposición de sodio y otros electrolitos como el potasio, cloruro, magnesio, entre otros, ya que estos regulan la presión osmótica celular y evitan el daño celular por hiponatremia.

La Tabla 2 presenta las diferencias entre distintos tipos de líquidos de venta en nuestro país que podrían beneficiar la reposición iónica.

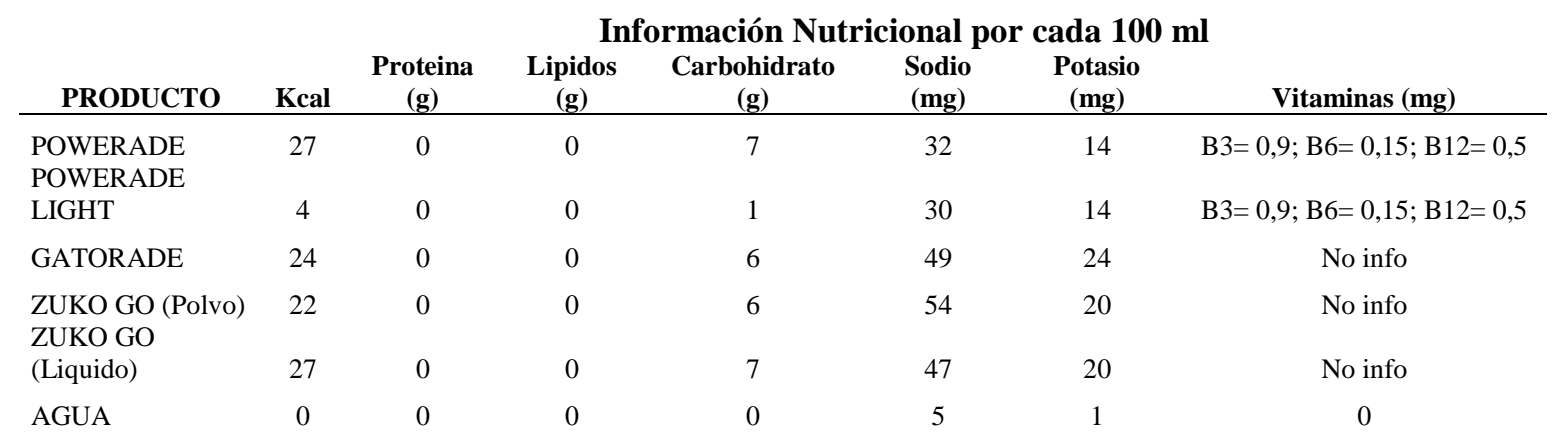

Tabla 2: Macronutrientes y micronutrientes contenidos en los distintos tipos de bebidas deportivas de venta común en nuestro país.

Este estudio muestra que casi dos tercios de los sujetos se hidrata sólo con agua sin la reposición adecuada de sodio, la cual resulta fundamental. En un estudio donde se comparó la eficacia de la rehidratación de una bebida con un contenido moderadamente alto de sodio $(0,15 \mathrm{~g} / 100 \mathrm{ml})$ con una solución con un bajo contenido en sodio
$(0,07 \mathrm{~g} / 100)$, se encontró que la bebida con $0,15 \mathrm{~g} / 100 \mathrm{ml}$ de sodio era capaz de rehidratar más rápido que la bebida con un bajo contenido en sodio (16); ya que aumenta la retención de fluidos, mejora la reposición del volumen plasmático $(17,18)$ y aumenta la palatibilidad, es decir, la necesidad de consumir voluntariamente líquido (19). Se recomienda que la reposición de 
sodio sea la misma que se pierde a través del sudor durante el ejercicio (20).

Otro factor importante es que las bebidas deportivas que contienen valores suficientes de sodio para provocar beneficios, tienen adicionado hidratos de carbono para favorecer la reposición de energía, provoca un aumento de la osmoralidad intestinal para favorecer la absorción, lo que provoca una disminución del agua corporal, favoreciendo la deshidratación (21).

Del grupo encuestado, un alto porcentaje demuestra hidratarse al hacer ejercicio, lo que representa más que una necesidad, una conciencia de los beneficios que otorga la reposición hídrica para el rendimiento físico. También un $88 \%$ de los sujetos se hidrata durante el ejercicio (Figura 1) lo que provocaría una disminución de los efectos de la deshidratación, como la hipertermia o diferencias en la osmoralidad celular y sanguínea.

Finalmente, los sujetos se hidratan principalmente motivados por el mecanismo de la sed, cuando ya existe una pérdida importante de líquido que perjudica el rendimiento. No existe el conocimiento adecuado de los beneficios que otorga una hidratación oportuna o las posibilidades de hidratarse con líquidos isotónicos o enriquecidos con carbohidratos que favorece la disponibilidad de energía, prolongando el tiempo de ejecución del ejercicio.

\section{REFERENCIAS BIBLIOGRÁFICAS}

1. Pitts GC, Johnson RE, and Consolazio FC. Work in the heat as affected by intake of water, salt and glucose. Am J Physiol, 1944.142: 253-259.

2. Rothstein A and Towbin EJ. Blood circulation and temperature of men dehydrating in the heat. In: Physiology of Man in the Desert, edited by Adolph EF. New York: Interscience, 1947, p. 172-196.

3. Gopinathan PM, Pichan G, and Sharma VM. Role of dehydration in heat stress-induced variations in mental performance. Arch Environ Health, 1988. 43: 15-17.

4. Sawka MN. Physiological consequences of hypohydration: exercise performance and thermoregulation. Med Sci Sports Exerc, 1992, 24: 657-670.

5. Naghii MR. The significance of water in sport and weight control. Nutrition Health, 2000, 14 (2):127-32.

6. Hubbard RW and Armstrong LE. The heat illnesses: biochemical, ultrastructural, and fluid-electrolyte considerations. In: Human Performance Physiology and Environmental Medicine at Terrestrial Extremes, edited by Pandolf KB, Sawka MN, and Gonzales RR. Indianapolis, IN: Benchmark, 1988, p. 305-359.

7. Coyle E. Fluid and carbohydrate replacement during exercise. How Much and Why? Sports Science Exchange. 1994. 7 (3); 13.

8. Boudou P, Fiet J, Laureaux C, Patricot MC, Guezennec CY, Foglietti MJ, Villette JM, Friemel F, and Haag JC. Changes in several plasma and urinary components in marathon runners. Ann Biol Clin, 1987. 45:37-45.

9. Barr S.I. Effects ok dehydration on exercise performance. Canadian Journal Applied Physiology. 1999. 24: 164-72.

10. Williams M. Nutrition for heath, fitness and sport. Ed. McGraw-Hill 2005.

11. Gisolfi C. Fluid balance for optimal performance. Nutrition Reviews, 1996.54 : 159-68.

12. Maughan R, Nadel E. Temperature regulation and fluid and electrolyte balance. In 
Nutrition in Sport, ed. R.J. Maughan 2000. Oxford: Blackwell Science.

13. Sanders B. Water and electrolyte shifts with partial fluid replacement during exercise. European Journal of Applied Physiology. 1999. 80: 318-23.

14. Mao IF, Chen ML, Ko YC: Electrolyte loss in sweat and iodine deficiency in a hot environment. Arch Environ Health, 2001.56:271-277.

15. Sharp, R. L. Role of Sodium in Fluid Homeostasis with Exercise J Am Coll Nutr 2006 25: 231S-239.

16. Rosés JM, Pujol P. Hidratación y ejercicio físico. Medicina de l'Esport. 2006;41:70-7.

17. Ray M, Bryan M, Ruden T, Baier S, Sharp $\mathrm{R}$, King D. Effect of sodium in a rehydration beverage when consumed as a fluid or meal J Appl Physiol, 1998. 85: 1329-1336.

18. Shirreffs SM, Taylor AJ, Leiper JB, Maughan RJ: Post-exercise rehydration in man: effects of volume consumed and drink sodium content. Med Sci Sports Exerc 1996, 28:12601271.

19. Minehan MR, Riley MD, Burke LM: Effect of flavor and awareness of kilojoule content of drinks on preference and fluid balance in team sports. Int J Sport Nutr Exerc Metab 2002, 12:81-92.

20. Maughan RJ and Leiper JB. Sodium intake and post-exercise rehydration in man. Eur $\boldsymbol{J}$ Appl Physiol 71: 311-319, 1995.

21. Gisolfi CV, Summers RW, Schedl HP, Bleiler TL, Oppliger RA: Human intestinal water absorption: direct vs. indirect measurements. Am J Physiol 1990, 258:G216222.

\begin{abstract}
The hydration in exercise is indispensable, this action can modify the physical performance due to the fact that the liquid consumption favors the thermoregulation. The lack of ingestion of fluids harms the maintenance of the corporal central temperature, harming the cardiovascular and cognitive function and, in some cases, provoking the death for dehydration or for the so called "heat blow". They were evaluated to 127 users of gymnasium of the commune of Viña del Mar, 39 women and 88 men. The subjects were voluntary that agreed to take part of the survey and that go between 3 at 5 times to the week to sessions of between 1 at 2 hours of exercise. The subjects are hydrated principally motivated by the thirst mechanism, when already there exists a important loss liquid that harms the performance. There does not exist the suitable knowledge of the benefits that grants an opportune hydration or the possibilities of being hydrated by liquids isotónics or high in carbohydrates that the availability of energy favors, prolonging the time of exercise
\end{abstract}

Key words: Hydration, Dehydration, Physical Activity.

Dirigir correspondencia a:

Mirta Crovetto Mattassi

Avenida Playa Ancha 850

Facultad de Ciencias Naturales y Exactas

Universidad de Playa Ancha

Chile.

mirtacrovetto@gmail.com

RECIBIDO 30 -7-2009

ACEPTADO 27-10-2009 